\title{
December 2013 Critical Care Case of the Month: I Don't Have a Drinking Problem
}

\author{
Robert Raschke MD \\ Elijah Poulos MD \\ Adam Bosak MD \\ Critical Care Medicine \\ Banner Good Samaritan Medical Center \\ Phoenix, AZ
}

\section{History of Present IIIness}

A 69-year-old male retired diabetic police officer was admitted to the ICU with intractable vomiting, severe abdominal pain and acute blindness. About a week prior, he suffered urinary frequency and was prescribed ciprofloxacin at urgent care with a presumptive diagnosis of urinary tract infection. Over the course of the week his urinary frequency resolved and he became anuric, he developed progressively worsening nausea and eventually vomiting to the point that he was unable to keep anything down, and severe bilateral lower abdominal and pelvic pain. His wife and son actually forced him into the ER when he became blind the day of admission. He denied fever, dysuria, cough and headache. In our emergency room he was noted to be in moderate distress with tachycardia, tachypnea, hyperpnoea and completely blind in both eyes unable to discern even simple shadows.

\section{$\mathrm{PMH}, \mathrm{SH}, \mathrm{FH}$}

The patient is a retired police officer with a past medical history of diabetes mellitus and benign prostatic hypertrophy. The patient denied alcohol, tobacco, or illicit drug use. He works out at a local gym almost daily since being diagnosed with diabetes a couple of years ago.

\section{Medications}

- Glipizide

- Metformin

- Tamsulosin

\section{Physical Exam}

Blood pressure $160 / 95 \mathrm{mmHg}$ with a heart rate of 110 , respiratory rate $35, \mathrm{SpO} 299 \%$ on $2 \mathrm{lpm}$ nasal cannula, and temp $36.0^{\circ} \mathrm{C}$. He appeared uncomfortable and moderately distressed, lethargic but arousable with GCS 13 . He was able to briefly answer simple questions. His eyes were conjugate, but did not track nor fix on objects placed in front of his eyes, and he could vaguely discern the light of a bright flashlight shined into both eyes. His pupils were 3-4 mm and fixed, with no light reflex elicitable, even with magnified examination of the pupil using an ophthalmoscope. On fundoscopic exam his discs were flat, and there were no hemorrhages or other lesions seen. He was tachycardic but regular with normal heart tones, and a bedside echocardiogram showed good left ventricular function. He had Kussmaul breathing with an odor of ketones and clear lungs. The lower abdomen was distended and tender, and a Foley catheter 
insertion returned 2 liters of yellow urine which resolved his abdominal pains. He had no peripheral edema and his hands were cool. The rest of his physical examination was unremarkable.

\section{Laboratory Evaluation}

Initial laboratory evaluation included a white blood count $24.3 \mathrm{~K} / \mathrm{mm} 3$ with $79 \%$ segmented neutrophils and no bands, hemoglobin $14.7 \mathrm{~g} / \mathrm{dL}$; sodium $138 \mathrm{mmol} / \mathrm{L}$; potassium $5.1 \mathrm{mmol} / \mathrm{L}$; chloride $92 \mathrm{mmol} / \mathrm{L}$; and $\mathrm{CO}_{2} 4 \mathrm{mmol} / \mathrm{L}$, yielding an anion gap of 44 when corrected. His BUN was $116 \mathrm{mg} / \mathrm{dL}$; creatinine of $7.7 \mathrm{mg} / \mathrm{dL}$. A venous blood gas showed a pH $6.77 \mathrm{pCO}_{2} 17 \mathrm{mmHg} ; \mathrm{pO}_{2} 73 \mathrm{mmHg}$; bicarbonate of $3 \mathrm{mmol} / \mathrm{L}$. Urinalysis showed negative leukocyte esterase, 1-5 leukocytes per HPF, glycosuria and ketonuria.

\section{Radiology Evaluation}

Admission chest $\mathrm{x}$-ray is in Figure 1.

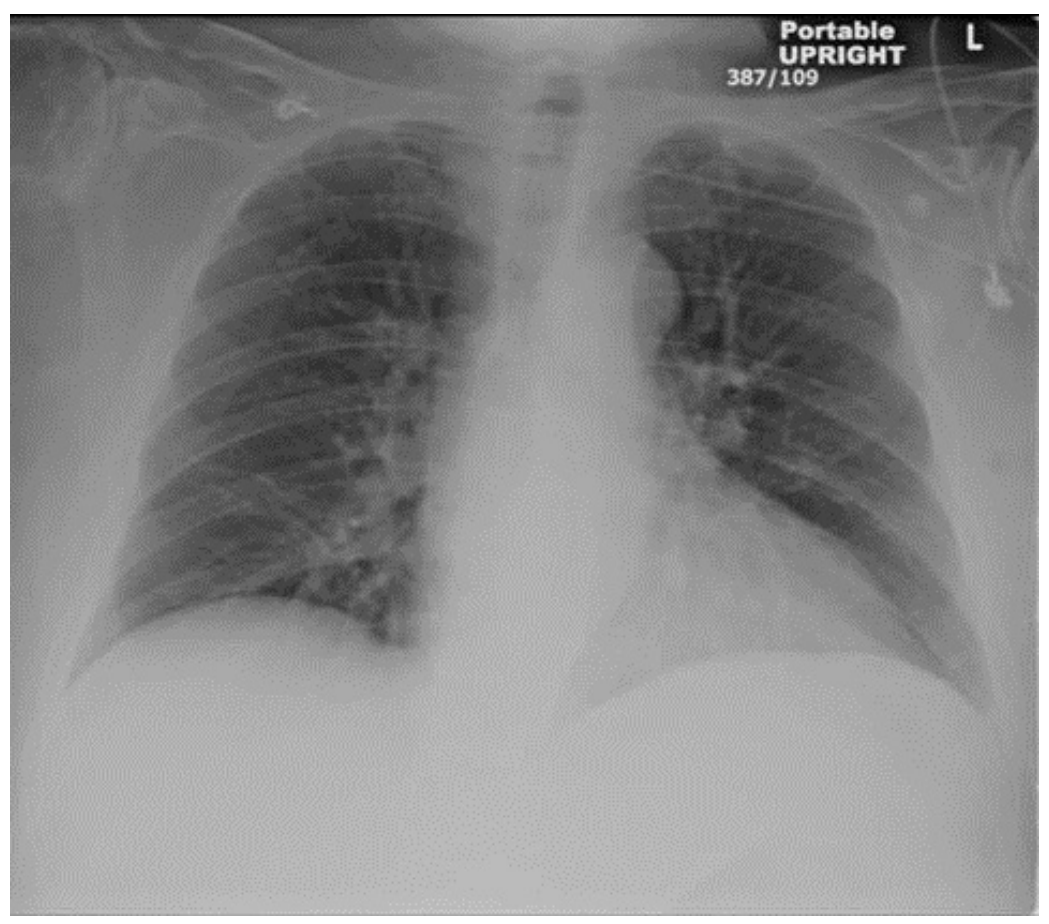

Figure 1. Admitting chest radiograph.

Computerized tomography of the abdomen showed no urinary tract obstruction (was performed after the Foley catheter was placed) and no other significant findings. Piperacillin/tazobactam and gentamicin were started for possible urinary tract infection with sepsis.

Which of the following is the best fits the clinical presentation explaining both his metabolic abnormalities and blindness?

1. Acute renal failure 
2. Alcoholic ketoacidosis

3. Diabetic ketoacidosis

4. Ethylene glycol ingestion

5. Methanol ingestion 


\section{Correct! \\ 5. Methanol ingestion}

The initial presentation mimicked a toxic alcohol ingestion. Methanol ingestion is a classic cause of anion gap metabolic acidosis and blindness - the acidosis predominantly due to formic acid, the oxidation metabolite of methanol. Ethylene glycol classically presents with acute renal failure and anion gap acidosis - the acidosis predominantly due to oxidative metabolites glycolic acid and oxalic acid. Diabetic ketoacidosis or alcoholic ketoacidosis usually does not present with blindness or this severe an acidosis. The patient certainly has acute renal failure from urinary tract obstruction but this is usually not associated with blindness.

Which of the following additional laboratory tests should be obtained?

1. Blood glucose

2. Lactic acid

3. Serum methanol

4. Urine for oxalate crystals

5. All of the above 


\section{Correct! \\ 5. All of the above}

Based on the diagnostic possibilities each of these should be obtained. His blood glucose was $127 \mathrm{~g} / \mathrm{dL}$. No methanol was detected in the serum. The urine was examined for oxalate crystals which are seen with ethylene glycol ingestion. None were found and no ethylene glycol was detected in the serum. His lactic acid level was 19.6 $\mathrm{mg} / \mathrm{dL}$. Additional laboratory values that returned negative included ASA, APAP and ethanol (consistent with his history of no substance use or abuse).

Based on this new information what is the most likely diagnosis?

1. Acute renal failure

2. Cardiogenic shock

3. Metformin-associated lactic acidosis (MALA)

4. Starvation ketosis

5. Starvation ketosis combined with acute renal failure 


\section{Correct! \\ 3. Metformin-associated lactic acidosis}

Type-B lactic acidosis occurs when no clinical evidence of poor tissue perfusion or poor oxygenation exists. He had a normal blood pressure and his ScvO2 $=79 \%$. We made tentative diagnoses of acute obstructive renal failure with dehydration leading to buildup of metformin and subsequent metformin-induced lactic acidosis, and likely starvation ketoacidosis. Two articles describing transient blindness due to MALA were identified in the medical literature $(1,2)$.

Which of the following is (are) true regarding therapy for MALA?

1. Hemodialysis should be performed as soon as possible for MALA

2. Sodium bicarbonate should be given immediately for MALA

3. There is no therapy proven to improve survival in MALA

4. $1+3$

5. All of the above 


\section{Correct! \\ 3. There is no therapy proven to improve survival in MALA}

In the absence of acute overdose, Metformin-associated lactic acidosis rarely develops in patients without comorbidities such as renal or hepatic insufficiency or acute infection. A systematic review found the incidence of such lactic acidosis to be fewer than 5.1 cases per 100,000 patient years and a mortality rate $>40 \%$ (3).

Supportive therapy is recommended with bicarbonate infusions to achieve $\mathrm{pH}>7.1$ followed by high-flux hemodialysis for both acidosis correction and drug removal, but there is no proven therapy which has been shown to improve survival in MALA (3). The mechanism of metformin-associated lactic acidosis is complex. Metformin normally works to decrease plasma glucose levels by inhibiting gluconeogenesis in the liver, decreasing intestinal glucose absorption and promotes the conversion of glucose to lactate in the splanchnic bed, and facilitating glucose uptake into myocytes by increasing insulin sensitivity.

When metformin levels become toxic, metformin may poison (inhibit) the cytochrome chain halting electron transport and oxidative phosphorylation, with an increase in the redox potential (NADH/NAD+). When oxidative phosphorylation stops, NADH cannot regenerate NAD+ and the Kreb's cycle winds down forcing pyruvate into lactate which uses up the abundance of NAD+ to drive the reaction. The excess lactate travels to the liver in the blood, which is supposed to reform pyruvate and send it into gluconeogenesis and the Kreb's cycle, but we already said the Kreb's cycle and gluconeogenesis has been shut down, so the lactate just continues to build up in the blood.

In healthy individuals, the excess lactate is simply cleared by other mechanisms (including uptake by the kidneys, when their function is unimpaired), and no significant elevation in blood levels of lactate occurs. When there is impaired renal function, however, clearance of metformin and lactate is reduced, leading to increased levels of both, and causing lactic acidosis with a buildup of lactic acid. Hemodialysis has been performed but survival in a small series was no different between those dialyzed and those not dialyzed (3).

In our patient the very low $\mathrm{pH}$ caused concern. A central line was placed and $300 \mathrm{mEq}$ $\mathrm{NaHCO}_{3}$ was given. A repeat venous blood gas showed a pH 7.06; $\mathrm{pCO}_{2} 20.5 \mathrm{mmHg}$; $\mathrm{pO}_{2} 59 \mathrm{mmHg}$; central venous $\mathrm{O} 2$ saturation $79 \%$, and another bolus of $100 \mathrm{mEq}$ of $\mathrm{NaHCO}_{3}$ was followed with an infusion at $50 \mathrm{mEq} / \mathrm{hr}$. The patient's blood pressure fell to $74 / 45 \mathrm{mmHg}$ and an intravenous infusion of levophed was started at $10 \mathrm{mcg} / \mathrm{min}$. High flux hemodialysis was started. Serum beta-hydroxybutyrate was $103 \mathrm{mg} / \mathrm{dL}$ (normal $<2.8 \mathrm{mg} / \mathrm{dL}$ ), and serum salicylate, ethanol, methanol and ethylene glycol levels were all undetectable.

Within four hours of arrival in the ICU, the patient was more alert and reported return of normal vision. His eyes tracked normally and demonstrated normal pupillary reflexes. 
An arterial blood gas drawn an hour later showed pH 7.24; $\mathrm{pCO}_{2} 16 \mathrm{mmHg}$ and bicarbonate of $6 \mathrm{mmol} / \mathrm{L}$. The consultant ophthalmologist subsequently arrived and documented a normal eye examination.

Sixteen hours after admission, hypotension and acidosis had completely resolved, and the urinary output was $100-200 \mathrm{~mL} / \mathrm{hr}$. Dialysis was discontinued. Cultures of urine and blood remained negative, and antibiotics were discontinued at 48 hours. The patient was discharged on the sixth day with normal renal function and a urology follow-up for benign prostatic hypertrophy. His metformin level eventually returned 9.4 (therapeutic range 1-2 and toxic levels producing lactic acidosis when $>5$ ).

\section{References}

1. Chu CK, Chang YT, Lee BJ, Hu SY, Hu WH, Yang DY. Metformin-associated lactic acidosis and acute renal failure in a type 2 diabetic patient. J Chin Med Assoc. 2003;66(8):505-8. [PubMed]

2. Kreshak AA, Clark RF. Transient vision loss in a patient with metformin-associated lactic acidosis. Am J Emerg Med. 2010;28(9):1059.e5-7.[CrossRef] [PubMed]

3. Peters N, Jay N, Barraud D, Cravoisy A, Nace L, Bollaert PE, Gibot S. Metforminassociated lactic acidosis in an intensive care unit. Crit Care. 2008;12(6):R149. [CrossRef] [PubMed] 